\title{
IoT based Edge Computing
}

\author{
Malti Bansal ${ }^{1 *}$ Harshit $^{2}$ \\ ${ }^{1}$ Department of Electronics \& Communication Engineering, Delhi Technological University (DTU), \\ Delhi-110042, India \\ ${ }^{2}$ Department of Mechanical Engineering, Delhi Technological University (DTU), Delhi-110042, India \\ E-mail: "
}

\begin{abstract}
Edge computing is a new way of calculating where most computer and storage devices are located on the internet, near mobile devices, sensors, end users, and internet of things devices. This physical approach improves delays, bandwidth, trust and survival.
\end{abstract}

Keywords: IoT, Edge Computing, bandwidth, Fog computing, Cloud

\section{INTRODUCTION}

Edge computing brings cloud power closer to the end or end user. There is a debate around edge computing vs fog computing. In fact, the two have the same goals. The slight difference is that using fog may involve using smart devices on a storage device and is more focused on Internet of Things (IoT). Telcom operators often manage the edge like mobile edge computing or multi-access edge computing- compute from the edge of the network. However, telco edge compute incorporates workloads that operate on customer machines and other presence areas on the customer site. We say that the edge of telco is better thought of as a distributed computer, operated by an operator, that can cross the border of the network and reach the edges of customers. Telco edge cloud incorporates the benefits of cloud and local computing, and 'local' can be on device or location. Customers can run low-latency applications, as well as cache or process data near a data source. This can also reduce the amount of background costs. Edge computing is an open source IT platform that enables systems to calculate data closer to or from a source rather than transferring data to the cloud. Edge computing enables real-time data processing without delay. The interaction of the digital world with people and objects through a communication network is defined as an edge. With the power of computing capabilities, systems can make data processing more efficient as large amounts of data can be processed in or near a source thus reducing internet bandwidth usage. Although the computer market on the edge is still young, the idea has been around for some time. According to IDC, "Edge computing is a network of small data centers that process or store sensitive data locally and compress all the data obtained in a data center or cloud storage, within an area of less than 100 square meters." During the Internet era, IT systems reverted to the same process where servers were installed to perform computer processes. Towards the end of 2005 businesses began using cloud computing for most of the fixes that occur in central data centers. Over the past few years, IoT and connectivity are on the rise, which has led to a renewed focus on computer infrastructure that recommends existing cloud computing systems.

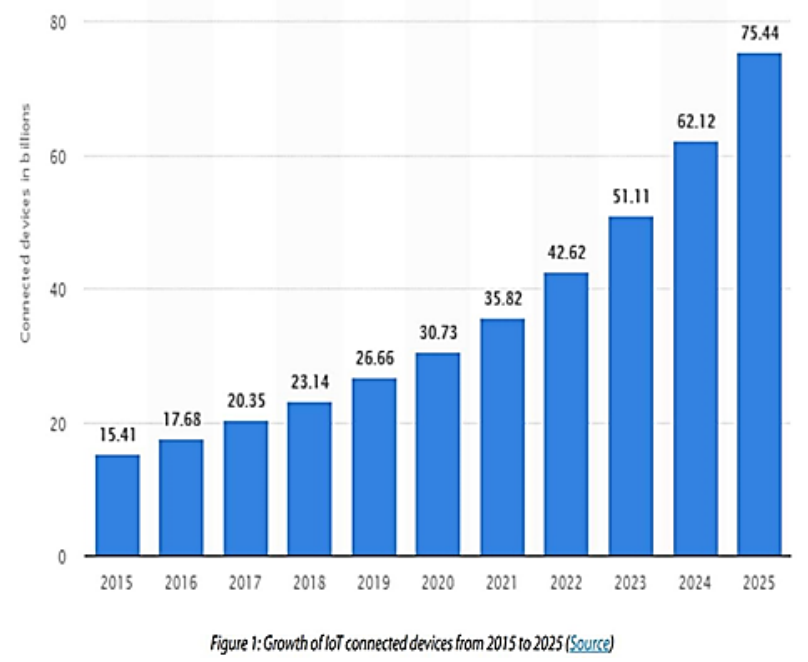

Edge computing is new way where third-party server resources are put on the Internet, next to mobile devices, sensors, end users, and the emerging IoT. Terms such as "cloudlets," "micro data centers," and "fog" have 
Journal of trends in Computer Science and Smart technology (TCSST) (2020)

Vol.02/ No. 04

Pages: 204-210

https://www.irojournals.com/tcsst/

DOI: https://doi.org/10.36548/jtcsst.2020.4.005

been used in literature to refer to these types of low-end computing hardware. They all represent the topic of integration and the big data centers that control speech in cloud computing. Edge computing refers to technologies that allow computing on the edge of a network, in the details below in the name of cloud services and in the top data in IoT services. "Edge" is said as any computer \& network services located on the path amongst data sources \& centers, as well as the continuation margin. Internet Border is a sole place. Typically only one stage gone from compatible end-to-end devices, offers a good set of low-latency substructure to provision developing submissions such as the unpopular genuineness of taxpayers we see, community security, linked and independent driving, intelligent production and health care. A great site for integrating, analyzing and deleting data bandwidth data on devices like photographic camera. New tasks and prospects rise as cloud computing integration happens the computer edge. Next, we explain what purposes can be done on the edge, and how edge computing hysterics into a modern computer model by introducing a standard three-dimensional model on the edge.

Three-Tier Edge Computing Model: We examine a few examples of independent edge computing applications. We present a standard model of three edge tier computers: IoT, edge, and cloud. The primary category is IoT, with buzzes, radars in a linked healthiness facility, devices and keen home appliances, and apparatus on the manufacturing Cyberspace. Many communication protocols are castoff to join the IoT and the subsequent tier, the edge. For instance, drones can link to a mobile tower via 4G / LTE, and smart home sensors can connect to the home gate via WiFi. Edge, with connected and private automobiles, cell tower, gate, and edge servers, requires significant computer power and cloud storage to complete complex tasks. Protocols amongst IoT and edge are typically characterized by less power and brief distance, while file protocols amongst the edge and the cloud have higher speeds and higher speeds. Ethernet, wireless cables, and the upcoming $5 \mathrm{G}$ are popular communication systems amongst the edge and the cloud.

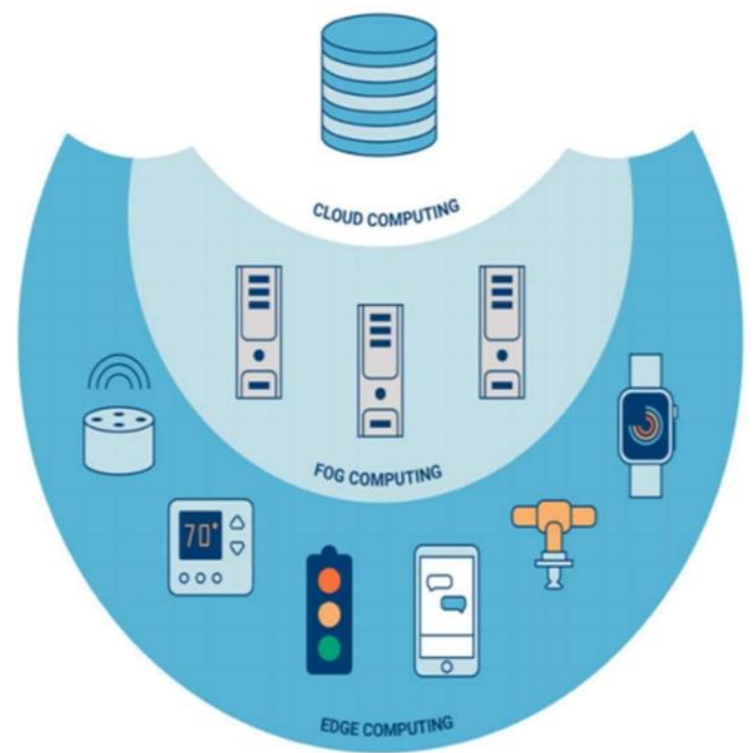

Fig.2: Edge Computing, Fog Computing and Cloud Computing

Fog Computing and how it is different from Edge Computing: Fog mist is defined as a state-of-the-art computer- based infrastructure that defines the best and most logical distribution of communication, computing and storage services between a data source and a computer. Fog computing was introduced by Cisco in 2014 where businesses can create duplicate items in line with the concept of using edge computing, in order to have better and more efficient computer performance. While in a computerized area on the edge computing takes place on the devices themselves, in fog computing occurs on a local network Data is transferred from devices to the gateway where it is transferred to a computer system and then back up.

\section{Components of Edge Computing:}

Infrastructure: Two major components of an edge computing infrastructure are computing infrastructure and data processing infrastructure. Currently, Bluetooth is the best way to connect power but it does not have the flexibility while mobile communication provides excellent compatibility but consumes a lot of power. 


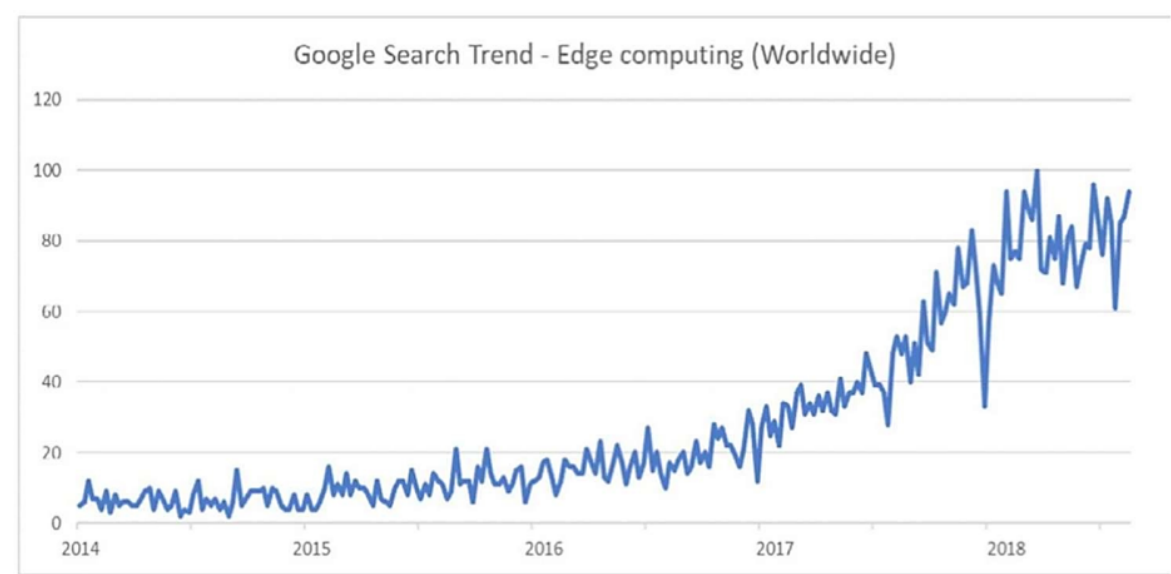

Fig.3: Google Search Trend--Edge Computing (Worldwide)

\section{Edge Computing Evolution:}

The edge computer industry has established speedily in current years since 2014. We practice edge computing as a keyword to hunt for the amount of articles printed annually on Google Scholar. Prior to 2015, edge computing was in the process of preparing for the technology. Since 2015, the amount of cutting-edge computers has increased ten-fold. Edge computing has arrived at a period of speedy development. Note that the amount of papers for 2019 is projected based on the results of the primary five months. We forecast that edge computing will continue to grow speedily until 2020. After 2020, edge computing will enter a period of consistent development. During this time, edge computing will see the amalgamation of professionals and industries, bring produce to business, and ultimately shape people's daily lives.

Beginnings of decentralized computing: The origins of edge computing can be drew back to the 1990s, at what time Akamai introduced a content delivery network (CDN). The impression back then was to launch nodes in areas close to the last user for the sending of saved content such as photos and videos. In 2001 awesome and widely dispersed claims used, as projected, various types of peers (called hash distribution tables) to cover networks. These coverage networks allow them to operate efficiently and tolerate errors, location, and load loading. In addition, these programs also make it likely to feat the network's closeness to sub-network communication, thus avoiding remote communication between nobles.

Cloud Computing: Cloud computing is a main factor in the past of edge computing and so earns superior mention. It involved some courtesy in 2006.This unlocked up a lot of new openings in terms of statistics, visibility, and storing volume. However, such cloud use was not the key to all usage cases. With the arrival of automotive and (industrial) IoT, for instance, there has been a cumulative stress on local information dispensation so that we can make faster decisions.

Fog Computing: In 2012, Cisco presented the word fog using a cloud-based infrastructure computer. The goal was to encourage IoT distribution, that is, to manage large amounts of IoT devices and large amounts of information for present low-latency applications.

Cloud and edge computing: Today, we see that in most cases, organizations opt for a combination of cloud and edge computing for complex IoT solutions. Cloud computing often works when organizations need storage and computing capacity to perform and process certain applications and processes, and to visualize telemetry data from anywhere. Edge computing, on the other hand, is a good choice in low latency, local autonomous actions, reduced backend traffic, and when private information is entered.

Edge Computing Present Applications: Edge computing is a method developed and distributed in cloud computing systems. Offering a number of benefits by eliminating processing data frequently in the cloud using network-based resources at the end of the network, it is very close to the data source. Edge resources offer technological advantages for cloud processing only and significantly improve system density while improving network effectiveness and dipping the number of round tours to the database.

Physical benefits based on edge devices improve real-time data statistics as well reduce internal access barriers used in actual programs such as machine learning and AR / VR. 
Journal of trends in Computer Science and Smart technology (TCSST) (2020)

Vol.02/ No. 04

Pages: 204-210

https://www.irojournals.com/tcsst/

DOI: https://doi.org/10.36548/jtcsst.2020.4.005

Oil and Gas Remote Monitoring: Monitoring of actual security is essential for serious structure and resources such as oil and gas. With this security and dependability in mind, numerous IoT nursing devices are still being industrialized to protect serious equipment and systems from disasters. Modern high-tech devices use the Internet of Things materials for heat, humidity, pressure, noise, humidity and radiation. Combined with the wide range of internet protocol enabled camera (IP cameras) and other technologies, this reveals a large and incessant quantity of data that is compiled and examined to deliver important information that can dependably assess the wellbeing of any application. Edge software allows data to be examined, treated and sent to end users in actual. It empowers control hubs to enter data as it happens, foresaw and prevent failures in a highly opportune manner. This is the very effective key, as period is of the spirit in these critical programs. This sounds very real when we face serious structures such as oil, gas and other energy services.

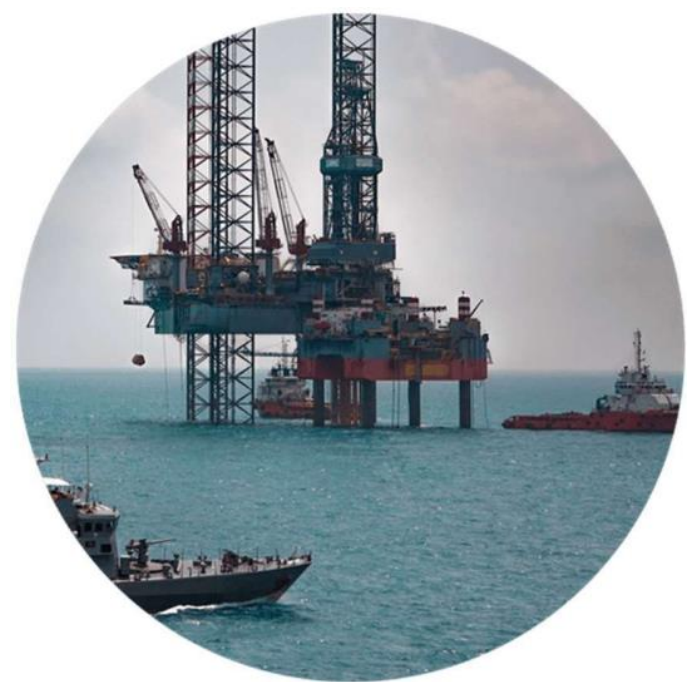

Fig.4: Oil and Gas Remote Monitoring

Edge Video Orchestration: Edge video orchestration utilizes edge computing capitals to tool a highly enhanced delivery technique for the extensively used yet bandwidth-heavy resource- video. In its place of delivering video from the central network to all the stages of the network, it logically edits, stores and allocates video files as near to the device as possible. Consider it suitable as a very effective and unique example of a video download network (CDN) just for the end users. Video MEC-enabled orchestration is very useful in big public areas. Stadiums, Concerts and other local events rely heavily on live video streaming and analytics to make and maximize income brooks. Newly created video clips and live streams can be used to quickly pay clienteles locally through ironic broadcasting dispensation applications that run on portable servers on the mobile edge and in their destination. This reduces the cost of the service and evades many superiority issues arising as of the conditions that become bottlenecks and TBs of video congestion hitting portable networks. This is somewhat $5 \mathrm{G}$ edge computing is intended to resolve in the approaching years. Presently, the EE operator is examining the possibility of such activities in partnership with Wembley Stadium, the UK's national football stadium.

Traffic Management: Owing to the extremely costly complexity of circulation management productivity, one of the finest ways to improve traffic management systems is to improve real-time data. Intellectual navigation systems make wide use of edge computing technology, particularly traffic management processes. Infection of IoT devices with large amounts of live data requires further dispensation and sifting near devices, earlier these thousands of data streams can hit the core / cloud networks. Using edge computing GBs of sensory and specialized data are analyzed, filtered and compressed before being transferred to the edges of IoT Gateways across several other operating systems. This considers the savings of network costs, storing and working costs of traffic management keys. 


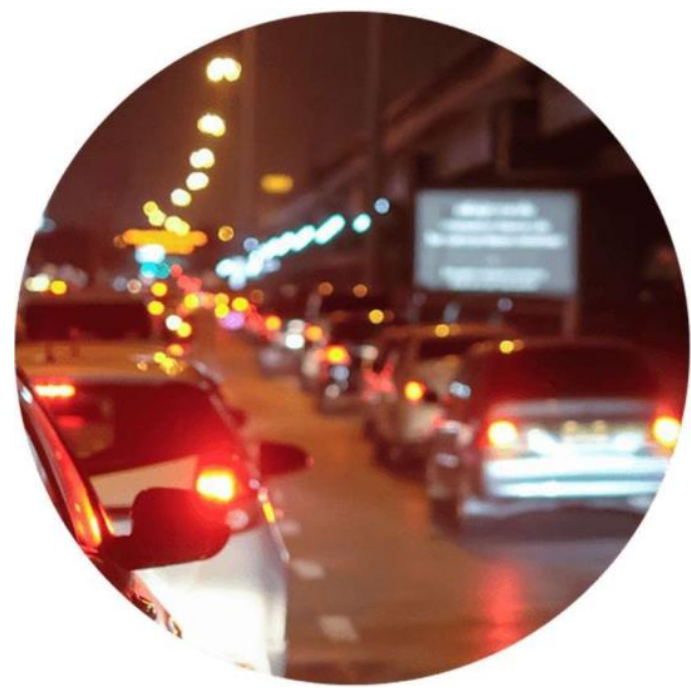

Fig.5: Traffic Management

Autonomous Vehicles: Though private cars are not yet ready for normal autonomous operation, deprived of computer technology, their performance could be less for many years to come. With the decline of moore's law and the advanced power of computing, on-board computers will now create huge costs for private cars. The vast majority of sophisticated sensory knowledge in private automobiles needs considerable bandwidth and real computing power. Edge techniques and computer distribution enhance security, location awareness and interaction with the next-generation. With mobile edge computing, cars can interchange real-time sensory data, adapt and advance decisions with fewer resources.

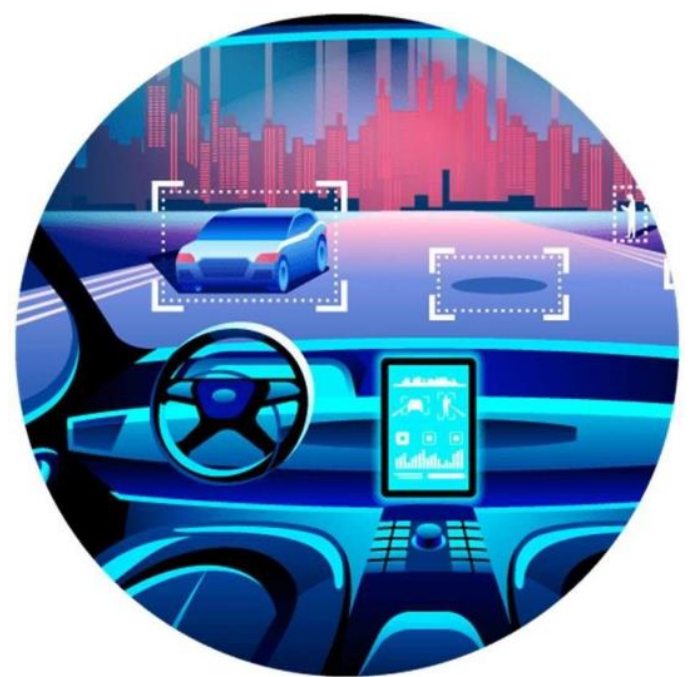

Fig.6: Autonomous Vehicles

Grid Edge Control and Analytics: Keen Grids, as we see, actually works by launching bilateral communication systems amid control distribution structure, receiver and the skull of state- the end. This is done by means of tested and certified broadband network (WAN) protocols. The unbelievable growing rate of the Internet of Things is slowly unraveling on the industrial side (IIoT), transporting with it many technologies that can monitor, achieve and regulate numerous activities in the power grid delivery structure. As the accelerated program is based on relic fuels in renewable energy, today's hard-working grids, nowadays tasked with adopting established technologies that are able to integrate and manage altogether these energy sources distributed across existing grids, form an integral network - grid clever. Edge grid computer technology empowers resources with progressive real-time monitoring capabilities and analytics abilities, making data more efficient and important for power-generating resources such as upgrades. This is something from solar roofs to solar farms for sale, electric vehicles, wind farms and power dams. Smart meters generate a large volume of valuable information that can support mathematical resources in power generation, availability, needs and high utilization predictions. This allows resources to indirectly avoid power outages and excessive compensation and reduce complete expenses and energy waste. For it to work, longwinded 
Journal of trends in Computer Science and Smart technology (TCSST) (2020)

Vol.02/ No. 04

Pages: 204-210

https://www.irojournals.com/tcsst/

DOI: https://doi.org/10.36548/jtcsst.2020.4.005

meter data needs to be treated from an internal basis for Grid Edge Controllers, compression and filtering data into critical, active packets past transmission over the service network.

Future of Edge Computing: Edge computing has arisen as a novel form of network planning that disregards outmoded computer issues, with some reports predicting its apparent growth in the approaching years. According to the report, the overall extent of the computer market is probable to grow from US \$2.8 billion by 2019 to US $\$ 9.0$ billion by 2024 , at the CAGR by 26.5 percent during the monsoon season. In terms of its short-range commitment, edge computing will see a fixed increase in its growing value and maturity crossways the industry by 2020 . The edge cloud facility market will grow by at least $50 \%$. Social media mega cloud providers, telecommunications companies, platform software providers, content delivery networks, and data center data providers set up to create basic services such as IaaS services and innovative cloud computing services on a computerized computer infrastructure. The goal of these is vendors provide IaaS and platform-as-a-service (PaaS) services that operate individualistically or with a central connection only to public assets and data center properties. By 2020, this new market will see explosive growth. Companies will choose multi-vendor bundled keys over sole vendors. Due to the range of bandwidth and connectivity restrictions, keeping computer platforms on edge will initiative organizations to work with collaborators to support their computer solutions rather than build and deploy their own. This applies to all industries including retail, manufacturing, transportation and operational services. According to a study by Forrester, major benefits organizations seeking to use edge computing include suppleness to address current and upcoming intelligence needs and that computing at the end evades network delays and lets for quicker responses. Besides, as noted by TechCrunch, Microsoft chief executive Satya Nadella recently said that the upcoming of the computer might be at an all-time high, where a computer is created in the area before data is moved to the cloud for machine learning purposes. To know the impact, it will have on the computer industry, one has to see the study, which forecasts that there will be 50 billion linked devices in the world by 2030, a figure she finds surprising, suggests Nadella. He added: "I mean this is very good. We think of billions of Windows devices or billions of smartphones. These are 50 billion [devices], and that is an average."

\section{CONCLUSION}

As IoT spreads widely, edge computing will do the same. The ability to analyze data near to the source will reduce delays, reduce online loads, improve privacy and security, and reduce data management costs. The cloud will continue to play a critical role in compiling important data and analysis in this massive set to harvest data that is no longer distributed back to edge devices. The combination of computer and cloud computing will help you better manage and analyze your data and greatly increase the value of your IoT efforts.

\section{REFERENCES}

[1] Introduction of the Edge Computing

a. Consortium. [Online].:

b. http://en.ecconsortium.org/

[2] Here's How Powerful All US Data

a. Centers Uses, Data Center

b. Know., San Francisco, CA, USA, 2016.

[3] NSF / Intel collaboration at ICN on

a. Wireless Edge Networks,

b. Nat. Science. Found., Alexandria, VA, USA, 2016.

[4] Introduction to Open Fog Consortium. [Online]. Available:

a. https://www.openfogconsortium.org/

[5] Automotive Edge Computing Consortium. [Online]. Available: https://aecc.org

a. Bio-IT World Edge Track. [Online]. Available: https://www.bio-itworldexpo.com/edge\#

[6] KubEetge - Framework for Kubernetes Native Edge Computing. [Online]. Available:

i. https://kubeedge.io/en/blog/cncf-sandboxannouncement /

[7] F. Bonomi, R. Milito, J. Zhu, and S.

a. Addepalli, "Compog fog and its role in the Internet of Things," Proc. First editor. MCC Workshop Mobile Cloud Comput., 2012, pages 13-16.

[8] UM.2013. [11] Y. C. Hu, M. Patel, D. Sabella,

a. N. Sprecher, and V. Young, "Mobile edge computing - A key technology towards 5G," ETSI, Sophia Antipolis, France, ETSI, White Paper 11, 2015, pp. 1-16, vol. 11, no. 
Journal of trends in Computer Science and Smart technology (TCSST) (2020)

Vol.02/ No. 04

Pages: 204-210

https://www.irojournals.com/tcsst/

DOI: https://doi.org/10.36548/jtcsst.2020.4.005

[9] R. LaMothe, "Edge computing," Pacific Northwest Nat. Lab., Richland, WA, USA, Tech. Rep., 2013. Accessed: Mar. 2014. [Online]. Available: https://mafiadoc.com/edge-computingpacificnorthwest-national-laboratory__59d648481723dd08e35b7b77.html

[10] J. Ravi, W. Shi, and C.-Z. Xu, "Personalized email management at network edges," IEEE Internet Comput., vol. 9, no. 2, pp. 54-60, Mar. 2005.

[11] M. Satyanarayanan, P. Bahl, R. Caceres, and N. Davies, "The case for VM-based cloudlets in mobile computing,” IEEE Pervasive Comput., vol. 8. no. 4, pp. 14- 23, Oct./Dec. 2009.

[12] M. Bansal and Priya, "Application Layer Protocols for Internet of Healthcare Things (IoHT)," 2020 Fourth International Conference on Inventive Systems and Control (ICISC), Coimbatore, India, 2020, pp. 369-376, doi: 10.1109/ICISC47916.2020.9171092.

[13] M. Bansal, Priya, "Performance Comparison of MQTT and CoAP Protocols in Different Simulation Environments". G. Ranganathan, J. Chen, A. Rocha (eds) Inventive Communication and Computational Technologies. Lecture Notes in Networks and Systems, vol. 145, pp. 549-560, Springer, Singapore, https://doi.org/10.1007/978-981-15-7345-3 47

[14] M.Bansal, Priya, "Machine Learning Perspective in VLSI Computer Aided Design at Different Abstraction Levels", $2^{\text {nd }}$ Int. Conf. on Mobile Computing and Sustainable Informatics (ICMCSI 2021), Tribhuvan University, Nepal, 2021.

[15] M.Bansal, A. Goyal, A. Choudhary, "Industrial Internet of Things (IIoT): A Vivid Perspective", $5^{\text {th }}$ Int. Conf. on Inventive Systems and Control (ICISC 2021), India, 2021.

[16] M. Bansal, V. Sirpal, M. K. Choudhary, "Advancing e-Government using Internet of Things", ”, $2^{\text {nd }}$ Int. Conf. on Mobile Computing and Sustainable Informatics (ICMCSI 2021), Tribhuvan University, Nepal, 2021.

[17] CISCO The Internet of Things How the Next Evolution of the Internet Is Changing Everything White Pap (2011)http://www.cisco.com/c/dam/enus/about/ac79/docs/innov/IoT_IBSG_041 1FINAL.pdf

[18] M. Symeonides, D. Trihinas, Z. Georgiou,

a. G. Pallis, and M. Dikaiakos, "Query- driven descriptive analytics for IoT and edge computing," in Proc. IEEE Int. Conf. Cloud Eng. (IC2E), Jun. 2019, pp. 1-11.

[19] Vakali and G. Pallis, “Content delivery networks: Status and trends," IEEE Internet Comput., vol. 7, no. 6, pp.68-74, Nov. 2003, doi: 10.1109/ MIC.2003.1250586. 